\title{
Superoxide Dismutase Reduces Creatinine and NGAL by Restoring Oxidative Balance during Sepsis
}

\author{
Jufitriani Ismy ${ }^{1,2}$, Maimun Syukri ${ }^{3 *} \mathbb{0}$, Dessy R. Emril ${ }^{4}$, Nanan Sekarwana ${ }^{5}$, \\ Jufriady Ismy ${ }^{6} \mathbb{C}$ \\ ${ }^{I}$ Graduate School of Mathematics and Applied Sciences, Universitas Syiah Kuala, Banda Aceh, 23111, Indonesia. \\ ${ }_{2}^{2}$ Department of Pediatrics, Faculty of Medicine, Universitas Syiah Kuala, Banda Aceh, 23111, Indonesia. \\ ${ }^{3}$ Department of Internal Medicine, Universitas Syiah Kuala, Banda Aceh, 23111, Indonesia. \\ ${ }^{4}$ Department of Neurology, Faculty of Medicine, Universitas Syiah Kuala, Banda Aceh, 23111, Indonesia. \\ ${ }^{5}$ Department of Pediatrics, Faculty of Medicine, Universitas Islam Bandung, Bandung, 40161, Indonesia. \\ ${ }^{6}$ Departement of Surgery, Faculty of Medicine, Universitas Syiah Kuala, Banda Aceh 23111, Indonesia.
}

\begin{abstract}
Sepsis-associated overproduction of reactive oxygen species (ROS) and nitric oxide (NO) during pathogen infection leads to overwhelming oxidative stress, which has been recognized as a primary contributor to acute kidney injury (AKI). Hence, antioxidant therapy has been widely explored in order to find an effective treatment for sepsis-related AKI, in particular by using endogenous antioxidant - superoxide dismutase (SOD). We assessed the effect of oral SOD on the alteration of AKI biomarkers (creatinine and Neutrophil Gelatinase-Associated Lipocalin - NGAL) in endotoxininduced septic murine. The animals were assigned as a healthy control, a septic control, and three treatment groups (250, 500, and $1000 \mathrm{IU}$ oral SOD). Treatment of SOD was carried out by forcefeeding for 16 weeks prior to intraperitoneal injection of lipopolysaccharide (LPS). The sepsis was assessed using the murine sepsis score (MSS) after 12 hours post-LPS injection, where the changes in plasma SOD, ROS, NO, creatinine, and NGAL were measured by enzyme-linked immunosorben assay (ELISA). During sepsis, SOD was significantly decreased from its baseline level while other biomarkers were significantly increased $(\mathrm{p}<0.05)$ - except for NGAL. MSS exhibited a declining trend in SOD dosage-dependent manner, and was significantly different with that of septic control group at SOD dosage of $1000 \mathrm{IU}(\mathrm{p}<0.05)$. SOD treatment with a dosage as low as $250 \mathrm{IU}$ could prevent the abnormal expression of the tested biomarkers during sepsis. There were significant reduction of plasma ROS, NO, creatine and NGAL in rats treated with 1000 IU SOD. Our study suggests the protective effect of SOD against sepsis-induced AKI by scavenging ROS and NO.
\end{abstract}

\section{Keywords:}

Nitric Oxide (NO);

Sepsis;

Acute Kidney Injury;

Superoxide Dismutase

NGAL;

Oxidative Stress

\section{Article History:}

$\begin{array}{llll}\text { Received: } & 04 & \text { October } & 2021 \\ \text { Revised: } & 06 & \text { December } & 2021 \\ \text { Accepted: } & 23 & \text { December } & 2021 \\ \text { Available online: } & 27 & \text { February } & 2022\end{array}$

\section{1- Introduction}

Sepsis is a primary cause of acute kidney injury (AKI) and sepsis is more likely to cause AKI (26-50\%) than primary kidney diseases (7-10\%) in adults [1]. A multicenter study reported that sepsis was responsible for at least 50\% of AKI cases in intensive care units (ICUs) and was associated with high mortality [2]. A retrospective study of 11 years' duration suggested that sepsis was predominantly responsible for AKI in children admitted to hospitals [3]. Sepsis has been identified as an independent risk factor for the development of AKI [4], and AKI is a major contributor to the development of sepsis in critically ill patients [5-7].

\footnotetext{
${ }^{*}$ CONTACT: maimun_62@unsyiah.ac.id
}

DOI: http://dx.doi.org/10.28991/ESJ-2022-06-02-06

(C) 2022 by the authors. Licensee ESJ, Italy. This is an open access article under the terms and conditions of the Creative Commons Attribution (CC-BY) license (https://creativecommons.org/licenses/by/4.0/). 
The mechanism of sepsis-related AKI remains poorly understood. Evidence suggests that it may involve ischemiareperfusion (IR) injury in the glomerulus, nephron inflammation, hypoxia, and overproduction of oxidative stress, cytokines, and chemokines causing tubular injury and mesenchymal cell apoptosis [8,9]. Of these, oxidative stress could be considered as the most common mechanism. A postmortem study on humans with sepsis-related AKI revealed that renal tubular epithelial cell vacuolization was associated with oxidative stress [10]. The production of reactive oxygen species (ROS) and nitric oxide (NO) increased in septic patients, indicating the state of oxidative balance impairment [11]. Both ROS and NO, along with other inflammatory mediators, contribute to the pathogenesis of mitochondrial dysfunction [12-14]. Additionally, NO is also associated with renal hemodynamic alteration and tubular injury via nitric peroxide formation during sepsis [10].

In response to the generation of oxidative stress, endogenous superoxide dismutase (SOD) is synthesized in mitochondria with the main function of neutralizing free radicals $[15,16]$. However, a study found that SOD was significantly reduced in septic patients, leading to an antioxidant-oxidative stress imbalance [17]. To overcome this, studies have employed exogenous SOD to cure various oxidative stress-related diseases. A study using cats infected with feline immunodeficiency virus (FIV) reported that 30-days of administration of SOD improved the endogenous SOD and immune system [18]. Another study investigated the role of 12-days oral administration of melon-based SOD and found a significant reduction in diabetes-related intrarenal oxidative stress [19].

In the case of sepsis, the use of exogenous SOD as a therapeutic approach is still scarce. A study investigating septic murine model induced via cecal ligation puncture (CLP), revealed that an administration of SOD 2 hours prior to the sepsis induction showed improvement in plasma leukocytes, thrombocytes, lactate, and glucose; although the survival was not significantly different compared to control group [20]. The protective effect of SOD on lung tissue was reported by a study using a murine model with a similar CLP induction method [21]. Collectively, exogenous SOD could be considered a promising therapy for multi-organ injury caused by sepsis, including AKI.

The aim of this study is to investigate the effectiveness of melon-based SOD in managing oxidative stress as a treatment for sepsis-associated AKI. Herein, the murine model was injected with lipopolysaccharide (LPS) endotoxin, which is closely related to sepsis pathogenesis [22, 23]. The dosages of oral SOD were 250, 500, and 1000 IU for 16 weeks. The effect of SOD on sepsis-related AKI was assessed based on the expression of creatinine and neutrophil gelatinase-associated lipocalin (NGAL), the common biomarkers for early AKI.

\section{2- Materials and Methods}

\section{2-1- Materials}

The LPS, procured from Sigma-Aldrich (MO, USA), was isolated from Escherichia coli (serotype O55:B5). The LPS was used to induce sepsis in rat model. Commercial melon-based SOD (Glisodin $®$ ) used in this research was available in combination with gliadin. All the reagents used in this research were procured from Merck (Selangor, Malaysia).

\section{2-2-Study Design}

The research was conducted on murine model (Rattus norvegicus) which were obtained from Universitas Gadjah Mada (Yogyakarta, Indonesia). The LPS-induced sepsis rats were treated with commercial melon SOD through oral route administration for 16 weeks, where related biomarkers were measured before and post-treatment. The procedures employed in this research could be seen in Figure 1.

\section{2-3- Treatment and Sepsis Induction}

A total of $25 \mathrm{R}$. norvegicus weighing from 0.20 to $0.25 \mathrm{~kg}$ were firstly acclimated and then equally divided into five groups, namely group I, II, III, IV, and V. All groups received free access to food and water. During 16 weeks of treatment, rats in group III, IV, and V were fed with 250, 500, and 1000 IU SOD daily, respectively. Thereafter, to induce the sepsis, intraperitoneal injection of LPS (10 mg/kg body weight) were carried out for all groups, except for group I that was injected with saline (acting as healthy control). Group II was assigned as a septic control group which received only intraperitoneal injection of LPS without the administration of SOD. The venous blood was collected from each animal and the plasma were used to measure the expressions of SOD, ROS, NO, creatinine, and NGAL pre- and 12hours post-LPS injection. Sepsis in the murine model was assessed using Murine Sepsis Score (MSS) indicated by following parameters: physical appearance, eyes, response to stimuli, level of consciousness, breathing quality, and respiratory rate. The grading system for the MSS referred to the previous report [24].

\section{2-4-Biochemical Analysis}

Enzyme linked immunosorbent assays (ELISAs) were performed to determine the expressions of SOD, ROS, NO, creatinine, and NGAL using commercial Bioenzy ELISA kits (Jakarta, Indonesia). The serum samples (40 $\mu \mathrm{L})$ and streptavidin-horseradish peroxidase (HRP) $(50 \mu \mathrm{L})$ were sequentially added into a microwell that had been coated with rat antibody of each aforementioned biomarker. Samples were incubated for 60 min and washed five times with $0.35 \mathrm{~mL}$ Wash buffer in a microplate washer (ImmunoWash 1575, Jakarta, Indonesia) for $1 \mathrm{~min}$. Into each well, $50 \mu \mathrm{L}$ 
substrate A and 50 substrate B were sequentially added, followed by an incubation at $37^{\circ} \mathrm{C}$ for 10 min. Prior to the analysis using Bio Rad x Mark microplate spectrophotometer at $450 \mathrm{~nm}$, stop solution was added into each well (50 $\mu \mathrm{L})$.

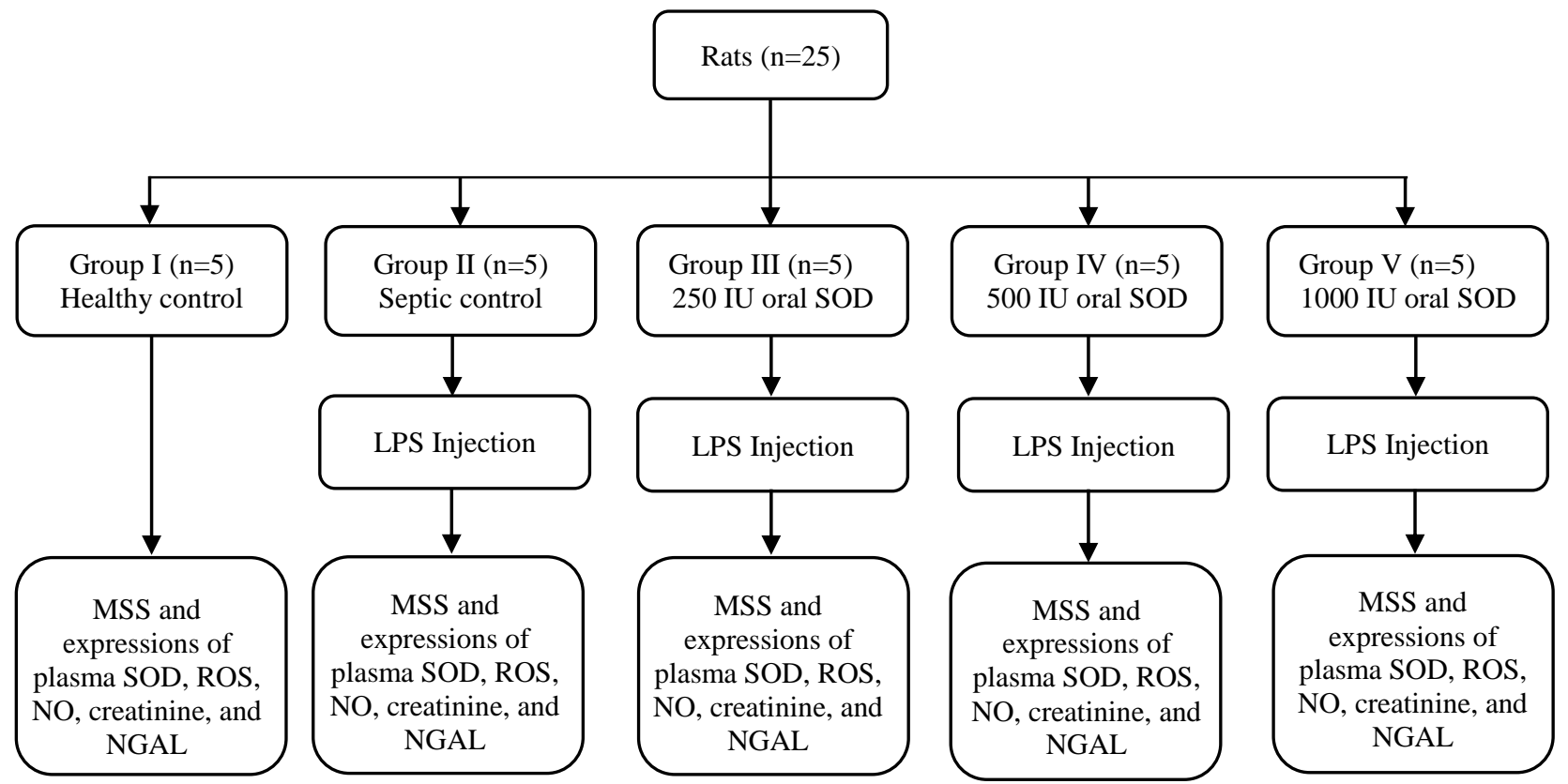

Figure 1. Flowchart of the procedures of study. The rats were equally divided into five groups, where the treated groups received oral SOD daily for 16 weeks. The MSS and expressions of plasma SOD, ROS, NO, creatinine, and NGAL were measured upon pre and 12-hour.

\section{2-5- Statistical Analysis}

MSS was statistically analyzed using Post Hoc Tamhane test. To compare the data of the markers pre- and postinjection of saline or PLS, the dependent t-test was used. To determine the between groups, analysis of variance (ANOVA) was employed.

\section{3- Results}

\section{3-1- Endotoxin-induced Sepsis}

Sepsis induction in rats were confirmed using MSS as presented in Figure 2. Group I which received saline injection only had no sign of sepsis. Compared to Group 1, those in Group II that received LPS injection only had higher MSS scores (mean $22.4 \pm 2.7)(\mathrm{p}<0.005)$. The MSS scores were declining in group I, II, and III treated with 250 , 500, and 1000 IU SOD, respectively. The reduction of sepsis scores was observed to be SOD dosage-dependent and significantly lower at $1000 \mathrm{IU}$.

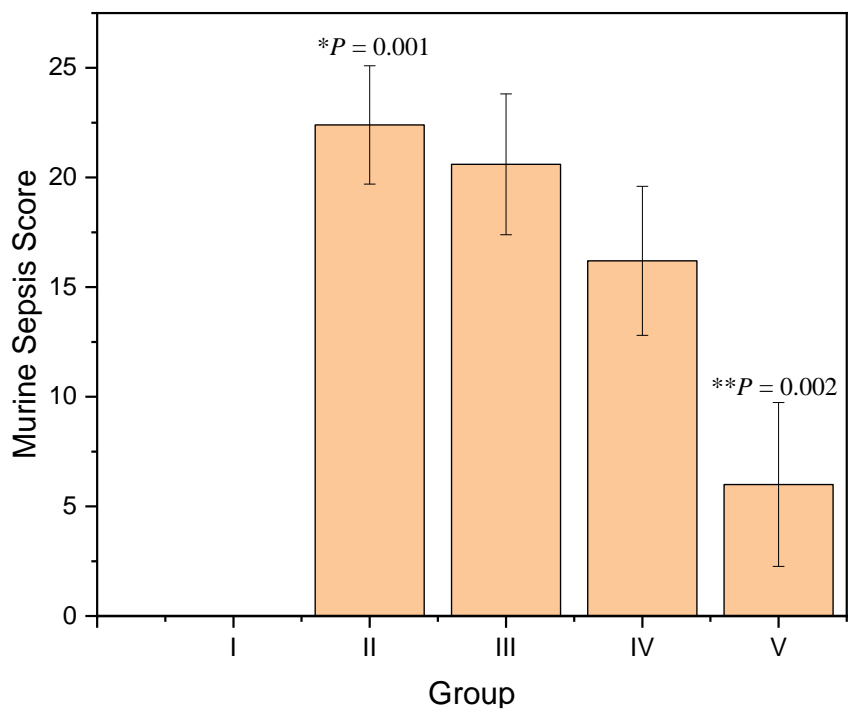

Figure 2. Murine sepsis score of samples in group I - V observed after 12 hours post-LPS injection. *Group II vs. group I and ** group II vs. group V; significant at $\mathbf{p}<0.05$ based on Post Hoc Tamhane test 


\section{3-2- Endogenous SOD}

The level of endogenous SOD post-LPS injection was significantly lower compared to pre-LPS injection suggesting SOD was affected by sepsis showing with $\mathrm{p}<0.05$ (Table 1). Similar level of endogenous SOD, however, could be maintained in a group with oral intake of 250 IU SOD. In groups administered with 500 and 1000 IU SOD, the depletion of SOD expression were 0.21-fold and 0.29-fold compared to baseline, respectively. However, the level of endogenous SOD in the aforementioned groups remained above the healthy control at 12 hours post-LPS injection. Additionally, a significant variation of endogenous SOD level before the LPS injection was also obtained, where the levels were higher in groups treated with oral SOD (Table 1).

Table 1. The effect of oral SOD on the expressions of SOD, ROS, and NO in LPS-induced sepsis rats

\begin{tabular}{|c|c|c|c|c|c|c|c|c|c|}
\hline \multirow{2}{*}{ Group } & \multicolumn{3}{|c|}{ Superoxide dismutase } & \multicolumn{3}{|c|}{ Reactive oxygen species } & \multicolumn{3}{|c|}{ Nitric oxide } \\
\hline & Pre-injection & Post-injection & $p^{a}$ & Pre-injection & Post-injection & $p^{a}$ & Pre-injection & Post-injection & $p^{a}$ \\
\hline Healthy control & $0.52 \pm 0.10$ & $0.55 \pm 0.14$ & 0.73 & $52.74 \pm 4.90$ & $51.50 \pm 7.16$ & 0.77 & $18.50 \pm 4.09$ & $18.16 \pm 2.22$ & 0.83 \\
\hline Septic Control & $0.60 \pm 0.00$ & $0.56 \pm 0.00$ & $0.00^{*}$ & $52.68 \pm 7.37$ & $62.18 \pm 6.16$ & $0.00^{*}$ & $17.91 \pm 1.54$ & $20.65 \pm 1.62$ & $0.03^{*}$ \\
\hline $250 \mathrm{IU}$ & $0.63 \pm 0.00$ & $0.73 \pm 0.10$ & 0.13 & $73.22 \pm 14.96$ & $68.32 \pm 5.90$ & 0.59 & $21.06 \pm 1.31$ & $19.92 \pm 2.36$ & 0.25 \\
\hline $500 \mathrm{IU}$ & $3.87 \pm 0.89$ & $0.83 \pm 0.00$ & $0.02^{*}$ & $64.50 \pm 8.34$ & $71.69 \pm 19.02$ & 0.50 & $18.14 \pm 2.30$ & $18.36 \pm 2.29$ & 0.91 \\
\hline $1000 \mathrm{IU}$ & $2.86 \pm 1.32$ & $0.83 \pm 0.14$ & $0.02^{*}$ & $64.20 \pm 7.22$ & $46.11 \pm 12.10$ & $0.00^{*}$ & $17.59 \pm 2.45$ & $16.08 \pm 3.56$ & 0.46 \\
\hline$p^{b}$ & $0.00^{*}$ & $0.00^{*}$ & & $0.01^{*}$ & $0.01^{*}$ & & 0.24 & 0.07 & \\
\hline
\end{tabular}

${ }^{*}$ Significant at $\mathrm{p}<0.05 ;{ }^{\mathrm{a}}$ Analyzed with dependent $t$-test; ${ }^{\mathrm{b}}$ Analyzed with ANOVA.

\section{3-3- ROS and NO}

Observation on plasma ROS and NO expressions suggested their significant increase upon 12 hours post-LPS injection at $\mathrm{p}<0.05$ (Table 1). Prior to the injection, the levels of ROS were significantly varied. The oral administration of SOD with a dosage of 250 and 500 IU were able of maintaining the similar value during sepsis. However, a significant decline of ROS as much as 0.72 -fold baseline was observed. As in the case of NO, there were no significant changes of its expression before and after the LPS injection at any SOD dosage (Table 1).

\section{3-4- Creatinine and NGAL}

The level of plasma creatinine, collected before and after LPS injection, were measured and the data are presented in Table 2. Our data suggested a dynamic change of creatinine level indicating by a significant reduction in healthy control. Meanwhile, its expression was significantly increase in plasma of septic rats without the treatment of oral SOD. The level was unchanged in a group treated with 250 IU SOD. Higher SOD dosage could yield a significant reduction of creatinine levels upon LPS injection. In contrast to creatinine, NGAL level did not change significantly during sepsis, although a certain extent of increment was observed. There was no significant different of NGAL level before and after LPS injection (Table 2). Nonetheless, daily SOD intake of at least 500 IU could significant reduced NGAL level upon sepsis. Plasma NGAL was also further reduced, reaching 0.59-fold baseline, by increasing the SOD dosage to 1000 IU.

Table 2. The effect of oral SOD on the expressions of creatinine and NGAL in LPS-induced sepsis rats

\begin{tabular}{ccccccc}
\hline \multirow{2}{*}{ Group } & \multicolumn{3}{c}{ Creatinine } & \multicolumn{3}{c}{ Neutrophil gelatinase-associated lipocalin } \\
\cline { 2 - 7 } & Pre-LPS & Post-LPS & $\boldsymbol{p}^{\boldsymbol{a}}$ & Pre-LPS & Post-LPS & $\boldsymbol{p}^{\boldsymbol{a}}$ \\
\hline Healthy control & $194.01 \pm 4.12$ & $187.73 \pm 5.63$ & $0.02^{*}$ & $20.25 \pm 4.5$ & $20.67 \pm 2.60$ & 0.56 \\
Septic control & $181.04 \pm 8.90$ & $191.15 \pm 7.53$ & $0.04^{*}$ & $18.11 \pm 2.86$ & $23.45 \pm 3.45$ & 0.06 \\
250 IU SOD & $193.11 \pm 10.68$ & $180.48 \pm 13.04$ & 0.11 & $22.32 \pm 3.97$ & $17.87 \pm 4.46$ & 0.07 \\
500 IU SOD & $196.95 \pm 11.92$ & $186 \pm 12.82$ & $0.01^{*}$ & $21.79 \pm 3.79$ & $17.67 \pm 3.64$ & $0.00^{*}$ \\
1000 IU SOD & $190.21 \pm 11.64$ & $176.69 \pm 9.78$ & $0.01^{*}$ & $21.58 \pm 3.96$ & $12.82 \pm 1.91$ & $0.01^{*}$ \\
$p^{b}$ & 0.15 & 0.21 & & 0.36 & $0.00^{*}$ & \\
\hline
\end{tabular}

${ }^{*}$ Significant at $p<0.05 ;{ }^{\text {a }}$ Analyzed with dependent $t$-test; ${ }^{\mathrm{b}}$ Analyzed with ANOVA

\section{4- Discussion}

In this present study, we observed the sepsis on rats after 12 hours post-LPS injection based on MSS score along with overwhelming oxidative stress. Previously, a study revealed that the intraperitoneal injection of LPS could induce sepsis in murine model that eventually led to AKI [23]. LPS administration causes systemic inflammation to the murine model by upregulation of proinflammatory cytokines [22]. As immune cells are activated, the inflammation could cause a 
mitochondrial dysfunction leading to the excessive release of ROS and NO. Additionally, hypoxic injury may occur during sepsis, leading to the overproduction of ROS via metabolic processes $[25,26]$.

Sepsis could progress to multi-organ dysfunctions, mostly associated with excessive oxidative stress [8]. Sepsis is the main contributor to the development of AKI thought several mechanisms such as glomerulus injury, inflammation, microvascular dysfunction, and tubular injury [8, 27]. Kidney acts as the main route for the elimination of amino acids metabolism product - creatinine. Patients with AKI have abnormalities in the excretion of urinary creatinine, therefore could be correlated with the severity of kidney failure [28]. Creatinine was also suggested as the most suitable biomarker for early diagnosis of sepsis-related AKI [29]. The significant increase on creatinine expression in our current observation, therefore suggests the initiation of renal function impairment. According to previous reports, significant increase of serum creatinine in human might occur after at least $50 \%$ of renal parenchyma has been injured [30, 31].

Furthermore, we conducted an observation on NGAL expression before and after sepsis induction using LPS injection. Its use as a biomarker of sepsis-induced AKI was based on the fact that NGAL production was stimulated by preventive measures of renal tubular epithelial cells against the ischemia-reperfusion injury [32]. Moreover, this biomarker has been reported more effective for early detection of sepsis-induced AKI, in comparison with creatinine [33]. However, our present results suggest NGAL expression in septic murine was not significantly different with the baseline. Nonetheless, previous in vivo study suggested the discriminative NGAL expression appeared optimum in plasma sample after 6 hours sepsis induction using LPS [34]. The difference in our findings with that of reported could be subjected to different the type of sample and analysis. The use of NGAL as the proposed biomarker is rather inconclusive, where different results were obtained from studies using different subjects, types of sample (urinary or serum), or hospital settings [35, 36].

Our findings showed that endogenous SOD in rats were suppressed upon LPS injection, disabling the recovery from oxidative imbalance. A previous prospective observation revealed significant attenuation of both plasma SOD and catalase activities along with their inverse correlation with the severity of organ failure [17]. SOD has a role in converting superoxide radical into hydrogen peroxide which could be eventually removed by catalase and peroxidase enzymes [37]. Hence, many have agreed on the therapeutic value of SOD to protect the mitochondria from oxidative damage during sepsis. To elevate the level of endogenous SOD, a study had employed intravenous infusion to administer exogenous SOD proved to improve the outcome in septic rats [38]. Induction of endogenous SOD had also already been carried out via subcutaneous injection of insulin resulting in improved outcomes [39].

Herein, the SOD administered through oral route enabled to increase the expression of endogenous SOD. Despite the injection of LPS, the SOD values in the treated groups were still higher than healthy control. It implicates that SOD elevation prior to sepsis onset could prevent the excessive oxidative stress as suggested by the observation on sepsis score that decreased in a concentration-dependent manner. It is further corroborated by significantly lower expressions of ROS and NO in plasma collected from SOD group, in contrast with septic control. The attenuation of oxidative stress subsequently reduces the risk of AKI as indicated by creatinine expression. Interestingly, daily intake of SOD as low as $250 \mathrm{IU}$ could maintain the level of creatinine upon sepsis. Moreover, increasing the SOD dosage could improve the creatinine clearance, where similar outcome was also reported in a randomized clinical trial using alkaline phosphatase therapy [40].

At 12 hours after LPS injection, NGAL expression was maintained in a group administered with 250 IU SOD. Further increase on the dosage led to significant drop of NGAL expression from the baseline. It is still unknown how SOD elevation could attenuate endogenous NGAL as triggered by sepsis induction. Currently, there is a debate on the role of NGAL in the progression of renal IR injury. One study suggested that NGAL provides protective effect against renal IR injury in murine model via autophagy activation [32]. Meanwhile, the other reported aggravated renal IR damage concomitant to NGAL-induced overproduction of autophagy in vitro [41]. Both, however, indicated the importance of NGAL in the pathogenesis of AKI, which could be potentially exploited for the therapeutic treatment. Hence, dosage of SOD should be carefully determined to yield the optimum outcome. It is also worth mentioning that the MSS was significantly lower in treatment group with 1000 IU SOD, in comparison with septic control group. In comparison with a published study who used tempol to induce endogenous SOD, they reported the effective dosage of 4-weeks 1.5 $\mathrm{mM} / \mathrm{kg} /$ day transdermal injection [42]. Other study required a dosage of $1 \mathrm{mmol} / \mathrm{L}$ oral tempol through drinking water for 8 weeks to achieve an improvement of oxidative balance in septic rats [43].

The strength of our investigation included the long duration of oral SOD administration to the rat sample with dosages ranged from $250 \mathrm{IU}$ to $1000 \mathrm{IU}$. Nevertheless, it is important to note the limitations in this study including the small size of the sample. In this case, heterogeneity among samples was hard to overcome. Furthermore, the observation of AKI occurrence in our study was only based on biomarkers. Creatinine has various clearance pathways making its use as a biomarker less definitive [44]. Similarly, NGAL is not only specifically produced in kidney tubules but also in failing myocardium which contributes to higher false-positive rate [45]. In the future, it is important to investigate the septic condition not only based on the biomarkers but also should be accompanied with histological analysis on the renal tissue. 


\section{5- Conclusion}

Administration of exogenous melon SOD to rat models could increase the level of the endogenous SOD, even following the intraperitoneal injection of LPS. The injection resulted in the occurrence of sepsis in a murine model based on MSS and changes in ROS and NO expression levels. Moreover, the increase in plasma ROS and NO is attributed to an increase in oxidative stress disruption, which is responsible for the development of AKI. Observation of the change in creatinine further suggests the initial stage of renal damage induced by a septic condition. However, NGAL expression was found to insignificantly increase after the LPS induction, which was probably due to the type of sample and analysis time. The role of SOD in improving the condition of septic murine could be observed in the MSS, where daily administration of 1000 UI SOD for 16 weeks was found to contribute significantly. Investigation into plasma ROS and NO revealed that administration of oral SOD had a role in preserving the oxidative balance, where the administration of SOD as low as 250 IU/day for 16 weeks was sufficient to keep the biomarker expressions insignificantly different from that of pre-LPS injection. A similar dosage of oral SOD was also found to yield an insignificantly different level of AKIassociated biomarkers (creatinine and NGAL). Taken altogether, the findings proved the protective effect of SOD against the development of AKI during sepsis by restoring the oxidative stress imbalance. Furthermore, our study suggested oral administration of SOD could manipulate the endogenous NGAL expression during sepsis, which could be a new treatment strategy for sepsis-related AKI. Nonetheless, further investigation needs to be carried out to confirm the role of exogenous SOD in the alteration of NGAL expression during sepsis.

\section{6- Declarations}

\section{6-1- Author Contributions}

Conceptualization, J.I.; validation, J.I., M.S., D.R.E., N.S., and Jy.I; formal analysis, J.I.; investigation, J.I; resources, J.I.; data curation, J.I.; writing — original draft preparation, J.I.; writing—review and editing, J.I., M.S., D.R.E., N.S., and Jy.I; supervision, M.S., D.R.E., and N.S. All authors have read and agreed to the published version of the manuscript.

\section{6-2- Data Availability Statement}

The data are not publicly available due to continuing study is ongoing. The data presented in this study are available on request from the corresponding author.

\section{6-3- Funding}

The authors received no financial support for the research, authorship, and/or publication of this article.

\section{6-4- Ethical Approval}

The protocol of this study was approved by the Committee Veterinary Ethics for Animal, Faculty of Veterinary Medicine, Universitas Syiah Kuala, Banda Aceh, Indonesia (No 20/KEPH/11/2019).

\section{6-5- Acknowledgements}

Authors would like to thank staff from Veterinary Medicine Lab, Universitas Syiah Kuala for the technical assistance.

\section{6-6- Conflicts of Interest}

The authors declare that there is no conflict of interests regarding the publication of this manuscript. In addition, the ethical issues, including plagiarism, informed consent, misconduct, data fabrication and/or falsification, double publication and/or submission, and redundancies have been completely observed by the authors.

\section{7- References}

[1] Kolhe, N. V., Stevens, P. E., Crowe, A. V., Lipkin, G. W., \& Harrison, D. A. (2008). Case mix, outcome and activity for patients with severe acute kidney injury during the first 24 hours after admission to an adult, general critical care unit: Application of predictive models from a secondary analysis of the ICNARC Case Mix Programme Database. Critical Care, 12(SUPPL. 1), 2. doi:10.1186/cc7003.

[2] Uchino, S., Kellum, J. A., Bellomo, R., Doig, G. S., Morimatsu, H., Morgera, S., Schetz, M., Tan, I., Bouman, C., Macedo, E., Gibney, N., Tolwani, A., \& Ronco, C. (2005). Acute renal failure in critically ill patients: A multinational, multicenter study. Journal of the American Medical Association, 294(7), 813-818. doi:10.1001/jama.294.7.813.

[3] Pundziene, B., Dobiliene, D., \& Rudaitis, Š. (2010). Acute kidney injury in pediatric patients: Experience of a single center during an 11-year period. Medicina, 46(8), 511-515. doi:10.3390/medicina46080073.

[4] Mehta, P., Sinha, A., Sami, A., Hari, P., Kalaivani, M., Gulati, A., Kabra, M., Kabra, S. K., Lodha, R., \& Bagga, A. (2012). Incidence of acute kidney injury in hospitalized children. Indian Pediatrics, 49(7), 537-542. doi:10.1007/s13312-012-0121-6. 
[5] Mehta, R. L., Bouchard, J., Soroko, S. B., Ikizler, T. A., Paganini, E. P., Chertow, G. M., \& Himmelfarb, J. (2011). Sepsis as a cause and consequence of acute kidney injury: Program to Improve Care in Acute Renal Disease. Intensive Care Medicine, 37(2), 241-248. doi:10.1007/s00134-010-2089-9.

[6] Shang, J., Wan, Y., Luo, C., Ye, G., Geng, Q., Auerbach, A., \& Li, F. (2020). Cell entry mechanisms of SARS-CoV-2. Proceedings of the National Academy of Sciences of the United States of America, 117(21), 11727-11734. doi:10.1073/pnas.2003138117.

[7] Manrique-Caballero, C. L., Del Rio-Pertuz, G., \& Gomez, H. (2021). Sepsis-Associated Acute Kidney Injury. Critical Care Clinics, 37(2), 279-301. doi:10.1016/j.ccc.2020.11.010.

[8] Peerapornratana, S., Manrique-Caballero, C. L., Gómez, H., \& Kellum, J. A. (2019). Acute kidney injury from sepsis: current concepts, epidemiology, pathophysiology, prevention and treatment. Kidney International, 96(5), $1083-1099$. doi:10.1016/j.kint.2019.05.026.

[9] Qian, J. Y., Wang, B., Lv, L. L., \& Liu, B. C. (2021). Pathogenesis of Acute Kidney Injury in Coronavirus Disease 2019. Frontiers in Physiology, 12, 586589. doi:10.3389/fphys.2021.586589.

[10] Aksu, U., Demirci, C., \& Ince, C. (2011). The pathogenesis of acute kidney injury and the toxic triangle of oxygen, reactive oxygen species and nitric oxide. Contributions to Nephrology, 174, 119-128. doi:10.1159/000329249.

[11] Hsiao, S. Y., Kung, C. Te, Su, C. M., Lai, Y. R., Huang, C. C., Tsai, N. W., Wang, H. C., Cheng, B. C., Su, Y. J., Lin, W. C., Chiang, Y. F., \& Lu, C. H. (2020). Impact of oxidative stress on treatment outcomes in adult patients with sepsis: A prospective study. Medicine, 99(26), e20872. doi:10.1097/MD.0000000000020872.

[12] Galley, H. F. (2011). Oxidative stress and mitochondrial dysfunction in sepsis. British Journal of Anaesthesia, 107(1), 57-64. doi:10.1093/bja/aer093.

[13] Rahbar Saadat, Y., Hosseiniyan Khatibi, S. M., Ardalan, M., Barzegari, A., \& Zununi Vahed, S. (2021). Molecular pathophysiology of acute kidney injury: The role of sirtuins and their interactions with other macromolecular players. Journal of Cellular Physiology, 236(5), 3257-3274. doi:10.1002/jcp.30084.

[14] Jin, L., Yu, B., Armando, I., \& Han, F. (2021). Mitochondrial DNA-Mediated Inflammation in Acute Kidney Injury and Chronic Kidney Disease. Oxidative Medicine and Cellular Longevity, 2021, 9985603. doi:10.1155/2021/9985603.

[15] Rosa, A. C., Corsi, D., Cavi, N., Bruni, N., \& Dosio, F. (2021). Superoxide dismutase administration: A review of proposed human uses. Molecules, 26(7), 1844. doi:10.3390/molecules26071844.

[16] Zarbock, A., Gomez, H., \& Kellum, J. A. (2014). Sepsis-induced acute kidney injury revisited: Pathophysiology, prevention and future therapies. Current Opinion in Critical Care, 20(6), 588-595. doi:10.1097/MCC.0000000000000153.

[17] Kumar, S., Gupta, E., Kaushik, S., Kumar Srivastava, V., Mehta, S. K., \& Jyoti, A. (2018). Evaluation of oxidative stress and antioxidant status: Correlation with the severity of sepsis. Scandinavian Journal of Immunology, 87(4), 12653. doi:10.1111/sji.12653.

[18] Webb, C. B., Lehman, T. L., \& McCord, K. W. (2008). Effects of an oral superoxide dismutase enzyme supplementation on indices of oxidative stress, proviral load, and CD4:CD8 ratios in asymptomatic FIV-infected cats. Journal of Feline Medicine and Surgery, 10(5), 423-430. doi:10.1016/j.jfms.2008.01.008.

[19] Naito, Y., Akagiri, S., Uchiyama, K., Kokura, S., Yoshida, N., Hasegawa, G., Nakamura, N., Ichikawa, H., Toyokuni, S., Ijichi, T., \& Yoshikawa, T. (2005). Reduction of diabetes-induced renal oxidative stress by a cantaloupe melon extract/gliadin biopolymers, oxykine, in mice. BioFactors, 23(2), 85-95. doi:10.1002/biof.5520230204.

[20] Warner, B. W., Hasselgren, P. O., James, J. H., Bialkowska, H., Rigel, D. F., Ogle, C., \& Fischer, J. E. (1987). Superoxide Dismutase in Rats with Sepsis: Effect on Survival Rate and Amino Acid Transport. Archives of Surgery, 122(10), $1142-1146$. doi:10.1001/archsurg.1987.01400220052010.

[21] Constantino, L., Gonçalves, R. C., Giombelli, V. R., Tomasi, C. D., Vuolo, F., Kist, L. W., de Oliveira, G. M. T., Pasquali, M. A. de B., Bogo, M. R., Mauad, T., Horn, A., Melo, K. V, Fernandes, C., Moreira, J. C. F., Ritter, C., \& Dal-Pizzol, F. (2014). Regulation of lung oxidative damage by endogenous superoxide dismutase in sepsis. Intensive Care Medicine Experimental, 2(1), 17. doi:10.1186/2197-425x-2-17.

[22] Copeland, S., Shaw Warren, H., Lowry, S. F., Galvano, S. E., \& Remick, D. (2005). Acute inflammatory response to endotoxin in mice and humans. Clinical and Diagnostic Laboratory Immunology, 12(1), 60-67. doi:10.1128/CDLI.12.1.60-67.2005.

[23] Jesmin, S., Gando, S., Zaedi, S., Prodhan, S. H., Sawamura, A., Miyauchi, T., Hiroe, M., \& Yamaguchi, N. (2009). Proteaseactivated receptor 2 blocking peptide counteracts endotoxin-induced inflammation and coagulation and ameliorates renal fibrin deposition in a rat model of acute renal failure. Shock, 32(6), 626-632. doi:10.1097/SHK.0b013e3181a5359c. 
[24] Shrum, B., Anantha, R. V., Xu, S. X., Donnelly, M., Haeryfar, S. M. M., McCormick, J. K., \& Mele, T. (2014). A robust scoring system to evaluate sepsis severity in an animal model. BMC Research Notes, 7(1), 233. doi:10.1186/1756-0500-7-233.

[25] Bugger, H., \& Pfeil, K. (2020). Mitochondrial ROS in myocardial ischemia reperfusion and remodeling. Biochimica et Biophysica Acta - Molecular Basis of Disease, 1866(7), 165768. doi:10.1016/j.bbadis.2020.165768.

[26] Kamis, F., Yegenaga, I., Musul, M., Baydemir, C., Bek, S., Kalender, B., \& Baykara, N. (2016). Neutrophil gelatinase-associated lipocalin levels during the first 48 hours of intensive care may indicate upcoming acute kidney injury. Journal of Critical Care, 34, 89-94. doi:10.1016/j.jcrc.2016.04.012.

[27] Anderberg, S. B., Luther, T., \& Frithiof, R. (2017). Physiological aspects of Toll-like receptor 4 activation in sepsis-induced acute kidney injury. Acta Physiologica, 219(3), 573-588. doi:10.1111/apha.12798.

[28] Di Micco, L., Quinn, R. R., Ronksley, P. E., Bellizzi, V., Lewin, A. M., Cianciaruso, B., \& Ravani, P. (2013). Urine creatinine excretion and clinical outcomes in CKD. Clinical Journal of the American Society of Nephrology, 8(11), $1877-1883$. doi:10.2215/CJN.01350213.

[29] Wang, K., Xie, S., Xiao, K., Yan, P., He, W., \& Xie, L. (2018). Biomarkers of sepsis-induced acute kidney injury. BioMed Research International, 2018, 6937947. doi:10.1155/2018/6937947.

[30] Bellomo, R., Kellum, J. A., \& Ronco, C. (2004). Defining acute renal failure: Physiological principles. Intensive Care Medicine, 30(1), 33-37. doi:10.1007/s00134-003-2078-3.

[31] Mishra, J., Dent, C., Tarabishi, R., Mitsnefes, M. M., Ma, Q., Kelly, C., Ruff, S. M., Zahedi, K., Shao, M., Bean, J., Mori, K., Barasch, J., \& Devarajan, P. (2005). Neutrophil gelatinase-associated lipocalin (NGAL) as a biomarker for acute renal injury after cardiac surgery. Lancet, 365(9466), 1231-1238. doi:10.1016/S0140-6736(05)74811-X.

[32] Zhang, Y. li, Qiao, S. kai, Wang, R. ying, \& Guo, X. Nan. (2018). NGAL attenuates renal ischemia/reperfusion injury through autophagy activation and apoptosis inhibition in rats. Chemico-Biological Interactions, 289, 40-46. doi:10.1016/j.cbi.2018.04.018.

[33] Kjeldsen, L., Johnsen, A. H., Sengelov, H., \& Borregaard, N. (1993). Isolation and primary structure of NGAL, a novel protein associated with human neutrophil gelatinase. Journal of Biological Chemistry, 268(14), 10425-10432. doi:10.1016/s00219258(18)82217-7.

[34] Han, M., Li, Y., Liu, M., Li, Y., \& Cong, B. (2012). Renal neutrophil gelatinase associated lipocalin expression in lipopolysaccharide-induced acute kidney injury in the rat. BMC Nephrology, 13(1), 25. doi:10.1186/1471-2369-13-25.

[35] Smertka, M., Wroblewska, J., Suchojad, A., Majcherczyk, M., Jadamus-Niebroj, D., Owsianka-Podlesny, T., Brzozowska, A., \& Maruniak-Chudek, I. (2014). Serum and urinary NGAL in septic newborns. BioMed Research International, $2014,717318$. doi:10.1155/2014/717318.

[36] Chang, W., Zhu, S., Pan, C., Xie, J. F., Liu, S. Q., Qiu, H. B., \& Yang, Y. (2018). Predictive utilities of neutrophil gelatinaseassociated lipocalin (NGAL) in severe sepsis. Clinica Chimica Acta, 481, 200-206. doi:10.1016/j.cca.2018.03.020.

[37] Pellegrino, D., La Russa, D., \& Marrone, A. (2019). Oxidative imbalance and kidney damage: New study perspectives from animal models to hospitalized patients. Antioxidants, 8(12), 8. doi:10.3390/antiox8120594.

[38] Macarthur, H., Couri, D. M., Wilken, G. H., Westfall, T. C., Lechner, A. J., Matuschak, G. M., Chen, Z., \& Salvemini, D. (2003). Modulation of serum cytokine levels by a novel superoxide dismutase mimetic, M40401, in an Escherichia coli model of septic shock: Correlation with preserved circulating catecholamines. Critical Care Medicine, 31(1), 237-245. doi:10.1097/00003246200301000-00037.

[39] Chen, G. D., Zhang, J. L., Chen, Y. T., Zhang, J. X., Wang, T., \& Zeng, Q. Y. (2018). Insulin alleviates mitochondrial oxidative stress involving upregulation of superoxide dismutase 2 and uncoupling protein 2 in septic acute kidney injury. Experimental and Therapeutic Medicine, 15(4), 3967-3975. doi:10.3892/etm.2018.5890.

[40] Pickkers, P., Heemskerk, S., Schouten, J., Laterre, P. F., Vincent, J. L., Beishuizen, A., Jorens, P. G., Spapen, H., Bulitta, M., Peters, W. H. M., \& van der Hoeven, J. G. (2012). Alkaline phosphatase for treatment of sepsis-induced acute kidney injury: A prospective randomized double-blind placebo-controlled trial. Critical Care, 16(1), 14. doi:10.1186/cc11159.

[41] Zhang, W., Yang, S., Cui, L., \& Zhang, J. (2016). Neutrophil gelatinase-associated lipocalin worsens ischemia/reperfusion damage of kidney cells by autophagy. Renal Failure, 38(7), 1136-1140. doi:10.3109/0886022X.2016.1158041.

[42] De Blasio, M. J., Ramalingam, A., Cao, A. H., Prakoso, D., Ye, J. M., Pickering, R., Watson, A. M. D., de Haan, J. B., Kaye, D. M., \& Ritchie, R. H. (2017). The superoxide dismutase mimetic tempol blunts diabetes-induced upregulation of NADPH oxidase and endoplasmic reticulum stress in a rat model of diabetic nephropathy. European Journal of Pharmacology, 807, 1220. doi:10.1016/j.ejphar.2017.04.026. 
[43] Cao, P., Ito, O., Ito, D., Rong, R., Zheng, Y., \& Kohzuki, M. (2020). Combination of Exercise Training and SOD Mimetic Tempol Enhances Upregulation of Nitric Oxide Synthase in the Kidney of Spontaneously Hypertensive Rats. International Journal of Hypertension, 2020, 2142740. doi:10.1155/2020/2142740.

[44] Lin, J., Fernandez, H., Shashaty, M. G. S., Negoianu, D., Testani, J. M., Berns, J. S., Parikh, C. R., \& Perry Wilson, F. (2015). False-positive rate of AKI using consensus creatinine-based criteria. Clinical Journal of the American Society of Nephrology, 10(10), 1723-1731. doi:10.2215/CJN.02430315.

[45] Lippi, G., \& Plebani, M. (2012). Neutrophil gelatinase-associated lipocalin (NGAL): The laboratory perspective. Clinical Chemistry and Laboratory Medicine, 50(9), 1483-1487. doi:10.1515/cclm-2012-0344. 